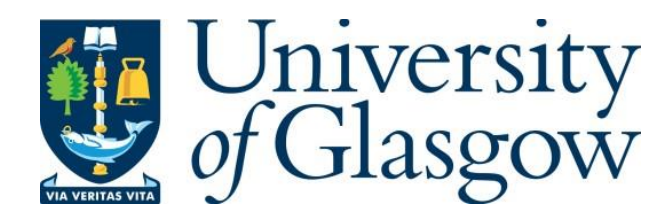

Lyu, Y., Yang, T., Lu, Z., Guo, C., Ge, C., Wang, Z., Jia, D. and Yin, H. (2017) External modulation method for generating accurate linear optical FMCW. IEEE Photonics Technology Letters, 29(18), pp. 1560-1563.

There may be differences between this version and the published version. You are advised to consult the publisher's version if you wish to cite from it.

http://eprints.gla.ac.uk/147704/

Deposited on: 11 September 2017

Enlighten - Research publications by members of the University of Glasgow http://eprints.gla.ac.uk 


\title{
External Modulation Method for Generating Accurate Linear Optical FMCW
}

\author{
Yingkai Lyu ${ }^{l}$, Tianxin Yang ${ }^{1}$, Zhaoyu $\mathrm{Lu}^{l}$, Cheng Guo ${ }^{l}$, Chunfeng Ge ${ }^{l}$, Zhaoying Wang ${ }^{l}$, Dongfang Jia ${ }^{l}$, \\ and Huabing Yin $^{2}$
}

\begin{abstract}
Frequency modulation continuous wave (FMCW) lasers are key components in modern optical imaging. However, current intracavity modulation lasers do not exhibit low frequency jitter rate and high linearity due to the inherent relaxation oscillations. Although this may be compensated in a direct modulation laser diode using an optoelectronic feedback loop, the available sweep speed is moderately small. In this work, a special external modulation method is developed to improve the performance of FMCW. Since only the first sideband optical field is used during the entire generation process, phase noise is kept to a minimum and is also independent of the sweep speed. We demonstrate that the linearity and jitter rates do not deteriorate appreciably when the sweep speed is changed over 3 orders of magnitude, even up to the highest sweep speed of $2.5 \mathrm{GHz} / \mu \mathrm{s}$.
\end{abstract}

Index Terms - Continuous-Wave Laser Radar, Linear Frequency Modulation, Laser Noise.

\section{INTRODUCTION}

$\mathrm{F}$ Trequency modulated continuous wave (FMCW) sources covering a broad frequency range have been used for a variety of optical imaging and sensor systems, such as light detection and ranging (LIDAR) [1-2], 3D imaging [3], optical coherence tomography (OCT) [4] and spectral analysis [5]. In these applications, high linearity, rapid sweep speed and low phase noise of FMCWs are critical. There has been extensive efforts made to update the characteristics of linear FMCWs, including enlarging sweep spans, increasing sweep speed or slope, reducing jitter rates, and improving linearity. Several methods based on intracavity modulation techniques have been reported for generating FMCWs, including Fourier Domain Mode Locking [6], semiconductor laser with a hybrid optoelectronic feedback loop [7-8], phase locking between two independent lasers [9], electronic-photonic heterogeneous integration [10], and optical heterodyne detection [11]. However, many of these suffer a common problem in terms of frequency jitter rates when the chirp rates reach several $\mathrm{GHz} / \mu \mathrm{s}$. This is due to the intrinsic relaxation oscillations in lasers together with other inherent side effects such as cavity mode

Manuscript received XX XX, XX; accepted XX XX, XX. Date of publication $\mathrm{XX} \mathrm{XX}, \mathrm{XX}$; date of current version XX XX, XX. This work was supported in part by the NSFC Project under Contract 61471256, Contract 61575143 and Contract 61377078.

${ }^{1}$ : College of Precision Instruments and Optoelectronic Engineering, Key Laboratory of Opto-Electronics Information and Technical Science, Tianjin University, Tianjin 300072, China. ${ }^{2}$ : School of Engineering, University of Glasgow, G12 8LT, UK. Corresponding author: Tianxin Yang, E-mail: tyang@tju.edu.cn). selection and mode competition inside the cavities. These effects result in the laser requiring a long time to reach a stable optical output after its frequency is changed. In practice, this means that jitter rates can become unacceptably high if the laser has not had enough time to reach a stable state when the frequency of the output light has been swept too fast (e.g. several $\mathrm{GHz} / \mu \mathrm{s})$. This can be seen in Wun's system [9] in which the frequency jitter rate increased by a factor of 6 when the sweep speed was increased by a factor of 6.5. This can mainly be attributed to the intrinsic relaxation oscillations present in lasers, especially unavoidable when they are modulated directly for generation of different linear sweep speeds. However, for many applications, high-quality, low jitter rate FMCWs with different linear sweep speeds are required. Ideally, low jitter rates should be maintained at a constant low level even when the linear sweep speed is varied over a few orders of magnitude.

In this paper, a new external modulation method to generate optical FMCWs with high quality is proposed and demonstrated experimentally. The jitter rate of our linear FMCW is largely independent of sweep speed and is constant as sweep speeds change over 3 orders of magnitude. This characteristic is ideal for ranging or imaging systems that require a large dynamic range of sweep speeds to balance scanning speed and resolution. In addition to these advantageous features, our system is simple and only employs one key device, called a single sideband (SSB) modulator. The extra-cavity SSB modulator, driven using a linearly swept electrical signal from an arbitrary waveform generator (AWG), plays the role of shifting, stepping or continuously varying the frequency of a continuous-wave (CW) beam emitting from a distributed feedback (DFB) laser. The frequency jitter rates and linearity (denoted as $R^{2}$ ) versus different sweep spans, as well as sweep speeds (or chirp rates) of the generated optical FMCWs, are measured and analyzed, respectively.

\section{PRINCIPLES AND EXPERIMENTAL SETUP}

\section{A. Principles of optical frequency shift}

To eliminate the relaxation oscillation effect, the intracavity modulations or direct modulations in lasers have to be replaced by external modulation. However, for normal optical intensity modulation, the phase noise in externally modulated optical waves still exists, causing jitter rates or frequency fluctuations that increase proportionally with the orders of sideband optical fields. For a free running continuous-wave laser diode $(\mathrm{CW}$ 


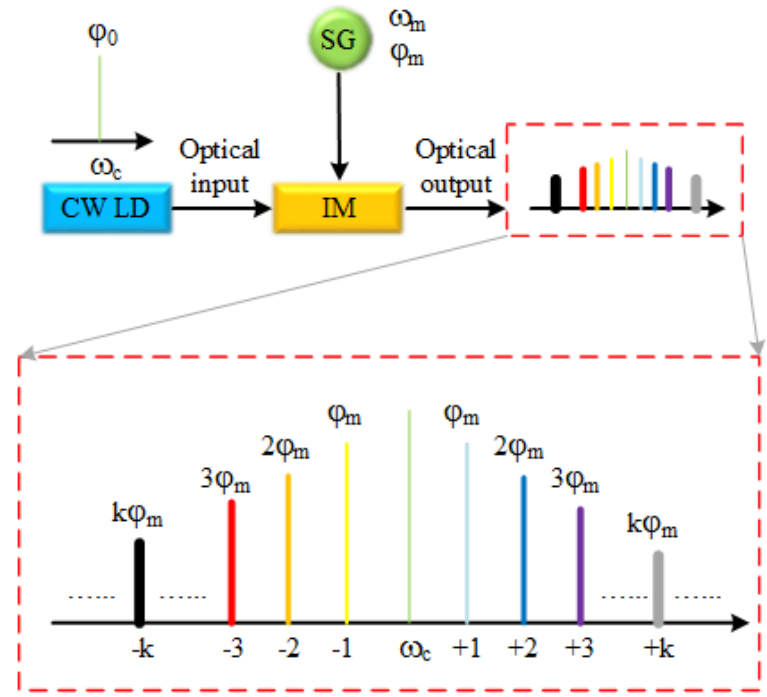

Fig. 1. The schematic phase noises of different orders of the sideband optical field in intensity-modulated CW LD light. CW LD: Free-running continuous-wave laser diode. IM: Intensity modulator. SG: Signal generator. The input light is fixed at $\omega_{c}$. SG generates a fixed value of phase noise $\left(\varphi_{m}\right)$ and frequency $\left(\omega_{m}\right)$. Close view shows the frequencies of the output light at different orders, where the width of each frequency indicates the amplitude of phase noise.

LD), intensity modulated by a sinusoidal signal with frequency $\omega_{m}$, the $k^{t h}$ order sideband optical field can be expressed by Eq. (1) shown below [12]:

$E_{k}(t)=E_{k} \exp \left\{-i\left[\left(\omega_{c}-k \times \omega_{m}\right) t+\varphi_{0}(t)+k \varphi_{m}(t)\right]\right\}(1)$

where $E_{k}$ is the $k^{\text {th }}$ order optical field amplitude, $\omega_{c}$ is center frequency of the CW LD, $\varphi_{0}(t)$ is phase noise of the CW LD, $\varphi_{m}(t)$ represents the phase noise resulting from the electrical signal generator. As shown in Fig. 1, the amount of phase noise is proportional to the order of the sideband optical field in Eq. (1). The first order sideband optical wave has minimum phase noise when employing external modulation. Based on this fact a SSB modulator was selected to be the frequency shift device as described previously [13]. This consists of a pair of parallel sub- Mach-Zehnder (MZ) interferometers (Fig. 2, MZI 1 and MZI 2). If an AWG delivers an electric signal with fixed frequency at $\omega_{m}$ in the radio frequency (RF) domain to drive the SSB modulator, along with appropriate bias voltages, the optical carrier wave at frequency $\omega_{c}$ and other sidebands can be effectively suppressed with the exception of the first order sideband optical field. As a result, the frequency of an incident optical wave at $\omega_{c}$ can be shifted to $\omega_{c}-\omega_{m}$ (Fig 2). By employing this mechanism, the device can be extended to shift the frequency of a CW LD continuously and linearly with time.

\section{B. Experimental Setup for optical frequency sweeps}

The experimental setup for the generation and detection of frequency linearly swept optical waves is illustrated in Fig. 3(a). The output CW light from a DFB laser (peak at $1552.824 \mathrm{~nm}$ ) with narrow linewidth of $\sim 50 \mathrm{kHz}$ was split into two paths by a $50 / 50$ coupler after a polarization controller (PC). The light in the upper path was modulated by a balanced SSB modulator

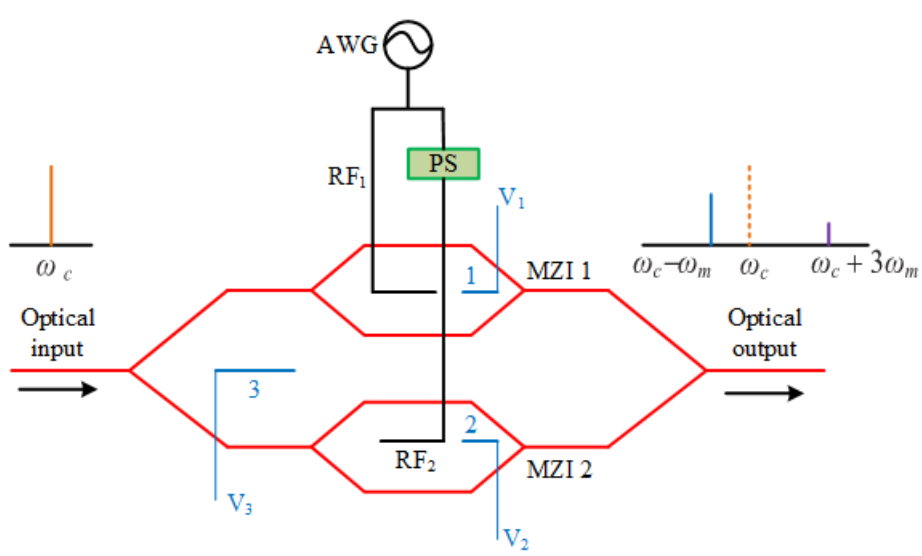

Fig. 2. Principle of frequency shift using a SSB optical modulator [13]. RF signals (black lines) generated by AWG together with biased voltages (blue lines) drive the SSB optical modulator (red lines), which shifts $\omega_{c}$ to $\omega_{c}-\omega_{m}$. PS: phase shifter; AWG: arbitrary waveform generator.

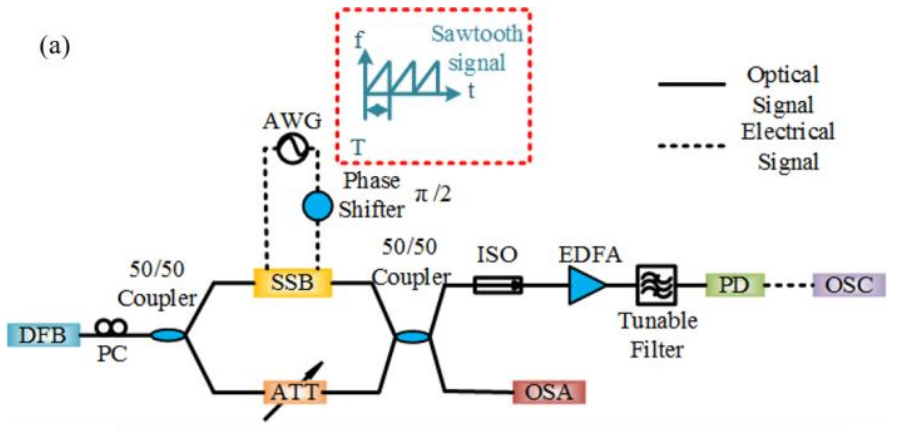

(b)

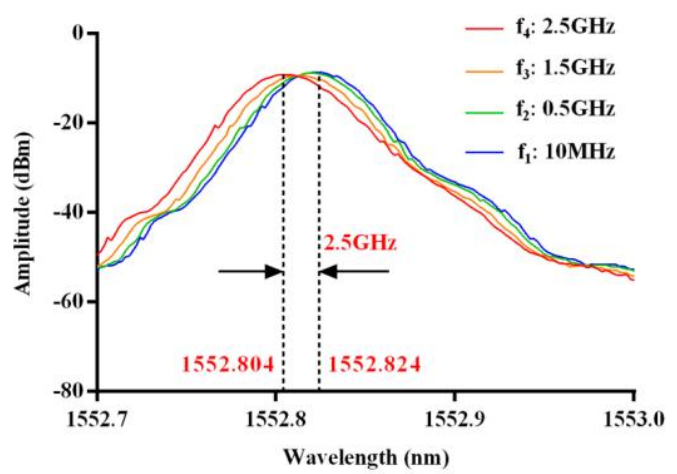

Fig. 3. (a) Experimental setup for the linearly swept light wave generation by an external modulation technique. The sawtooth signal in the red dashed box is generated by the AWG. (b) Four static frequency shifted spectrum curves indicate that the SSB modulator, driven by 4 fixed RF signals, has been worked in the state of optical frequency shifting. The maximum wavelength shifted from $1552.824 \mathrm{~nm}$ to $1552.804 \mathrm{~nm}$ which covers the sweep span of $2.5 \mathrm{GHz}$.

(Photline 6024-05), along with a phase shifter (Model 1831, KRYTAR), driven by an AWG (Keysight, M8190) with a maximum sampling rate of $12 \mathrm{GSa} / \mathrm{s}$. The current signal used to drive the SSB modulator was a linearly swept frequency signal generated from the AWG following a pre-programmed time-frequency pattern in the RF domain (i.e. a sawtooth signal) as shown in the inset of Fig. 3(a). The light power in the lower path was adjusted using an optical intensity variable attenuator (ATT) to maximize the beat signal of the recombined beams. After a fast photodetector (PD) (UPD-15-IR2-FC, bandwidth of 
$25 \mathrm{GHz}$ ), the frequency swept beat signal was observed using a real-time digital oscilloscope (OSC, Keysight, MSOV334A) at a maximum sampling rate of $80 \mathrm{GSa} / \mathrm{s}$ and a bandwidth of 33 GHz. The optical spectrum of frequency shifted light was monitored using an optical spectrum analyzer (OSA) to ensure that the SSB modulator was operating in the single sideband modulation mode ahead of the linearly frequency swept process starts. Four static frequency shifted spectrum curves in Fig. 3(b) indicate that the SSB modulator, driven by 4 fixed RF signals sequentially, has been worked in the state of optical frequency shifting.

When employing the frequency shift mechanism of a SSB modulator, linearly changing the frequency of the RF signal results in a linear sweep in the frequency of the light modulated by the SSB modulator. It should be noted that, in this approach, the maximum frequency sweep span and period of the RF signals can be set arbitrarily in the AWG. This is one advantage of this method. The most prominent advantage of this method is that the phase noise is always maintained at its minimum value and is independent of sweep as a consequence of the use of the first sideband optical field only during the entire modulation.
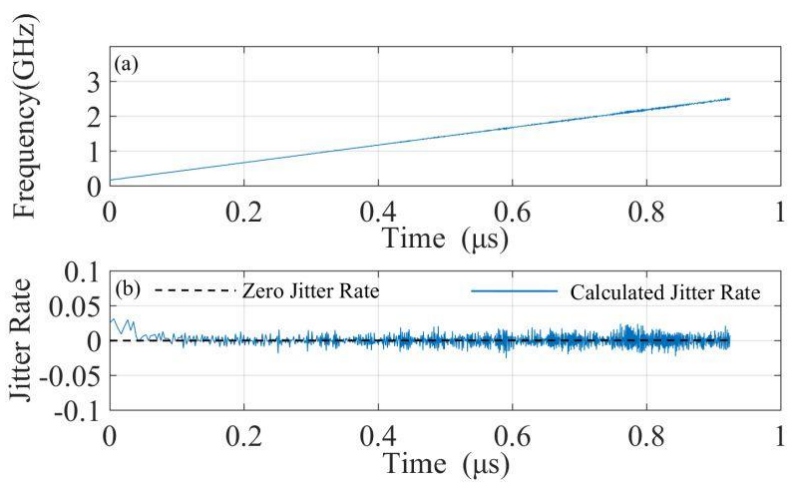

Fig. 4. (a) Electrical sawtooth signals from AWG which show linearly swept frequencies ranging from 0 to $2.5 \mathrm{GHz}$. (b) Jitter rates of the electrical signals presented in (a).
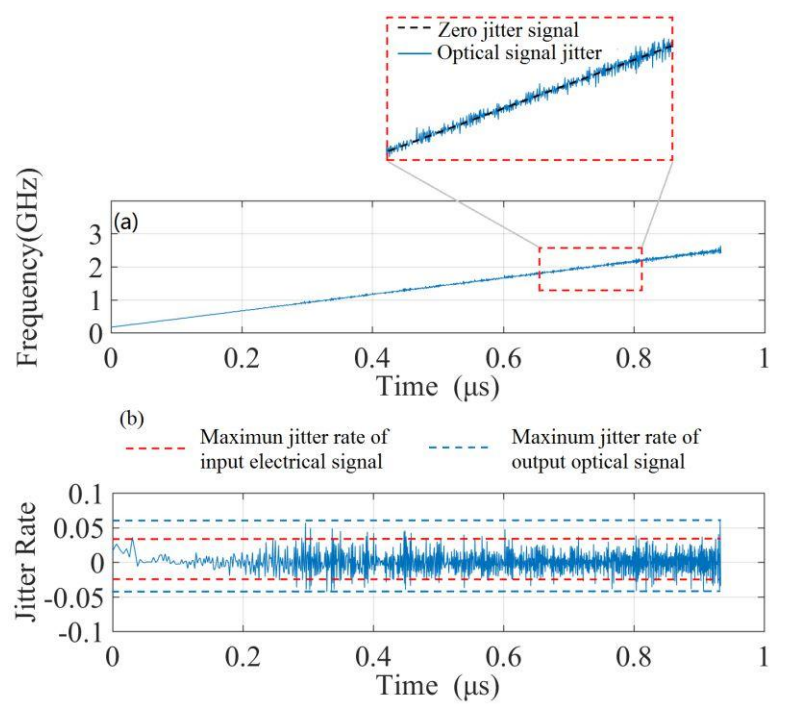

Fig. 5. (a) Linearly swept output optical frequencies ranging from 0 to $2.5 \mathrm{GHz}$. Close view in red dashed box shows the details of the linearity and jitter rates of the optical frequencies. (b) Jitter rates of the optical signals presented in (a). The dotted lines are reference lines for the maximum jitter rates for both electrical input and optical output signals.

\section{RESULTS AND DISCUSSIONS}

We first characterized the input electrical signals from the AWG. The linearity of frequency versus time was obtained via Fast Fourier Transformation of the raw data (Fig. 4a). To quantify the accuracy of the frequency, and jitter rates (Fig. 4b), the measured experimental data was fitting using an ideal line described by Eq. (2) shown below:

$$
\text { Jitter Rate }=\frac{f-f_{0}}{f_{0}}
$$

where $f$ is the instantaneous frequency of the measured experimental data while $f_{0}$ is the corresponding frequency value on the ideal straight line. Due to the limitation of the AWG itself, the electrical sawtooth signals used to modulate the SSB modulator were not ideal. This is reflected in its linearity of 0.9997 and the maximum jitter rate of $3.16 \%$ (Fig. 4). As shown in Fig. 5, the frequency variations of the output optical linearly swept signals are similar to that of the electrical sawtooth signals, with linearity and maximum jitter rate of 0.9990 and $5.69 \%$ respectively. This confirms the prediction that the first sideband optical field possesses the minimum phase noise, with most of the noise resulting from the electrical signals.

Using the same method, the linearity of the optical FMCW time-frequency curves at four different sweep speeds and their associated frequency jitter curves were obtained, as shown in Fig. 6. It was found that the linearity of electrical signals maintained 0.9997 at the four speeds (Fig. 6a). Although the linearity of the optical signals is slightly lower than that of the electrical input signals, it still reaches 0.9990 for sweep speeds ranging over 3 orders of magnitude. On the other hand, the jitter rates of the optical signals (Fig. 6b), fluctuate slightly from $5 \%$ to $8 \%$ when the sweep speed changes from $0.005 \mathrm{GHz} / \mu$ s to $2.5 \mathrm{GHz} / \mu \mathrm{s}$. This may, in part, be a consequence of the fluctuation of the input electrical signals generated by the AWG itself, which increases from $3 \%$ to $5 \%$. This shows that the jitter rates largely maintain the same level even though the sweep speed changes from $0.005 \mathrm{GHz} / \mu$ s to $2.5 \mathrm{GHz} / \mu$ s. This is
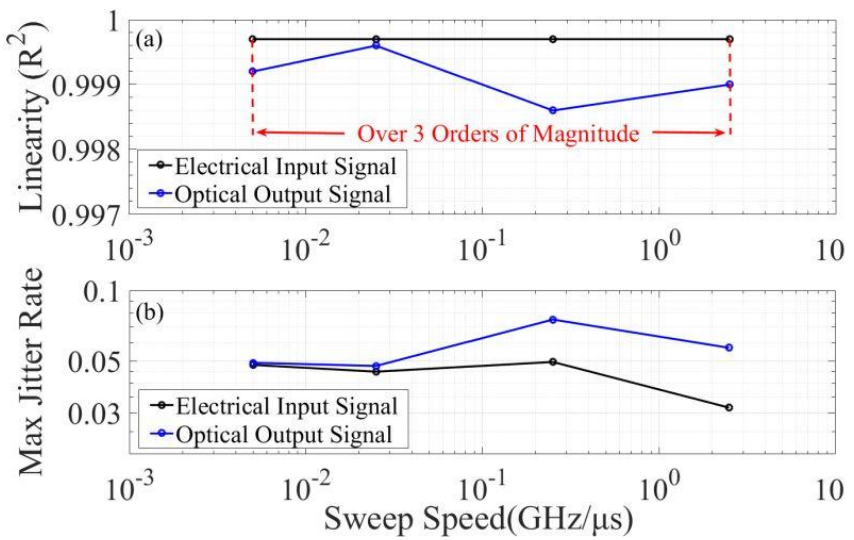

Fig. 6. (a) Linearity and (b) maximum jitter rates of both electrical and optical frequency linearly swept signals ranging from 0 to $2.5 \mathrm{GHz}$ at four different sweep speeds $(0.005 \mathrm{GHz} / \mu \mathrm{s}, 0.025 \mathrm{GHz} / \mu \mathrm{s}, 0.25 \mathrm{GHz} / \mu \mathrm{s}$, and $2.5 \mathrm{GHz} / \mu \mathrm{s}$ in sequence from left to right). 

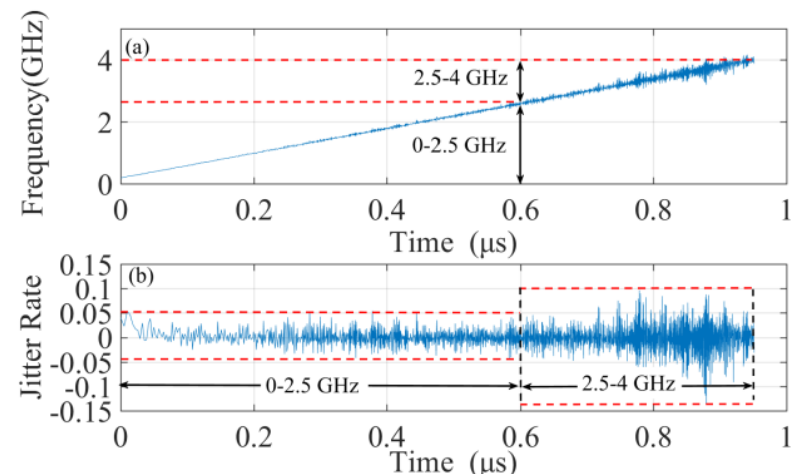

Fig. 7. (a) Sawtooth signals from the AWG which present the frequency of optical linearly swept signals ranging from 0 to $4 \mathrm{GHz}$. The red dotted lines indicate the frequency values of $2.5 \mathrm{GHz}$ and $4 \mathrm{GHz}$ respectively. (b) Jitter rate of the optical signals presented in (a). The red dotted lines indicate the maximum jitter rates in two different frequency spans respectively.

a significant improvement compared to the previous study [9] and demonstrates that the inference between the jitter rate and the sweep speed is substantially alleviated by employing the external modulation method. The result also implies that the relaxation oscillation in a laser cavity dominates and significantly affects the final accuracy of the optical FMCWs generated by internal modulation techniques. In contrast, using this external modulation technique, the effect of relaxation oscillations is completely avoided, and therefore permits the generation of high accuracy FMCW optical signals at high sweep speeds.

It is worth noting that, due to the limited sampling rate of the AWG used in our experiments ( $12 \mathrm{GSa} / \mathrm{s})$, the well-defined and acceptable frequency sweep span for an optical linearly swept signal was confined to the range of $0 \sim 2.5 \mathrm{GHz}$. Therefore, the accuracy of the optical swept frequencies begins to degrade as the sweep span increases up to $4 \mathrm{GHz}$, despite their high quality below $2.5 \mathrm{GHz}$ (Fig. 7). However, it is known that the sweep span could be extended to hundreds of gigahertz using a combination of multi-wavelength and synchronous filtering approaches. This will generate combined linearly swept output light with a bandwidth wide enough for application in demanding systems.

\section{CONCLUSION}

In conclusion, we present a simple external modulation method that generates high quality, continuous light with frequency that is linearly swept with time. Only the first sideband optical field is used in the system, resulting in a jitter rate that can be maintained at a low level when the sweep speed is varied over 3 orders of magnitude. The successfully generated linearly swept light can have the linearity of 0.9990 and the jitter rate of $5.69 \%$ over a span of $2.5 \mathrm{GHz}$ at an equivalent sweep speed of $2.5 \mathrm{GHz} / \mu \mathrm{s}$.

\section{References}

[1] C. V. Poulton, D. B. Cole, A. Yaacobi, and M. R. Watts, (2016). "Frequency-modulated Continuous-wave LIDAR Module in Silicon Photonics." Presented at Optical Fiber Communication Conference, OSA Technical Digest (online) (Optical Society of America), paper W4E.3.
[2] C. R. Doerr, L. Chen, T. Nielsen, R. Aroca, L. Chen, M. Banaee, S. Azemati, G. McBrien, S. Y. Park, J. Geyer, B. Guan, B. Mikkelsen, C. Rasmussen, M. Givhechi, Z. Wang, B. Potsaid, H. Lee, E. Swanson, and J. Fujimoto, (2016). "O, E, S, C, and L Band Silicon Photonics Coherent Modulator/Receiver." Presented at Optical Fiber Communication Conference Postdeadline Papers, OSA Technical Digest (online), paper Th5C.4.

[3] K. B. Cooper, R. J. Dengler, N. Llombart, T. Bryllert, G. Chattopadhyay, E. Schlecht, J. Gill, C. Lee, A. Skalare, I. Mehdi, and P. H. Siegel, (2008). "Penetrating 3-D imaging at 4- and 25-m range using a submillimeter-wave radar," IEEE Trans. Microw. Theory Tech. 56(12), 2771-2778.

[4] Lee, H. D., Jeong, M. Y., Kim, C. S., \& Shin, J. G. (2014). "Linearly wavenumber-swept active mode locking short-cavity fiber laser for in-vivo oct imaging." IEEE Journal of Selected Topics in Quantum Electronics, 20(5), 1-8.B.

[5] Szafraniec, A. Lee, W. I. Mcalexander, R. D. Pering, T. S. Tan, and D. M. Baney, (2004). "Swept coherent optical spectrum analysis," IEEE Transactions on Instrumentation and Measurement, 53(1), 203-215.

[6] R. Huber, M. Wojtkowski, and J. G. Fujimoto, (2006). "Fourier Domain Mode Locking (FDML): A new laser operating regime and applications for optical coherence tomography." [J]. Optics Express, 14(8):3225-37.

[7] N. Satyan, A. Vasilyev, G. A. Rakuljic, V. Leyva, and A. Yariv, (2009). "Precise control of broadband frequency chirps using optoelectronic feedback," Optics Express, 17(18), 15991-15999.

[8] N. Satyan, A. Vasilyev, G. A. Rakuljic, J. O. White, and A. Yariv, (2012). "Phase-locking and coherent power combining of broadband linearly chirped optical waves," Optics Express, 20(23), 25213-25227.

[9] J. Wun, C. Wei, J. Chen, C. S. Goh, S. Y. Set, and J. Shi, (2013). "Photonic chirped radio-frequency generator with ultra-fast sweeping rate and ultra-wide sweeping range," Optics Express, 21(9), 11475-11481.

[10] N. Quack et al., "Development of a FMCW LADAR Source Chip Using MEMS-Electronic-Photonic Heterogeneous Integration", (2014). GOMACTech Charleston SC USA, March 31 - April 3.

[11] Z. W. Barber, F. R. Giorgetta, P. A. Roos, I. Coddington, J. R. Dahl, R. R. Reibel, N. Greenfield and N. R. Newbury, (2011). "Characterization of an actively linearized ultrabroadband chirped laser with a fiber-laser optical frequency comb," Optics Letters, 36(7), 1152-1154.

[12] Ishizawa, A., Nishikawa, T., Goto, T., Hitachi, K., Sogawa, T., \& Gotoh, H. (2016). "Ultralow-phase-noise millimetre-wave signal generator assisted with an electro-optics-modulator-based optical frequency comb". Scientific Reports, 6, 24621.

[13] Z. Wang, X. Qin, C. Gao, T. Wang, D. Jia, M. Sang, and T. Yang, (2013). "Precise Simultaneous Multiwavelength Tuning by Electrical RF Signals," IEEE Photonics Technology Letters, 25(10), 914-916. 